\title{
SIMULATIONS OF SUPERLUMINAL RADIO SOURCES
}

\author{
S.S. KOMISSAROV ${ }^{1,2}$, S.A.E.G. FALLE ${ }^{1}$ \\ ${ }^{1}$ Department of Applied Mathematics, The University of Leeds, \\ Leeds LS2 9JT \\ ${ }^{2}$ Astrospace Centre, Lebedev Physical Institute, 53, Leninsky \\ Prospect, Moscow B-333, 177924 Russia
}

The superluminal knots of VLBI-sources are widely believed to be shocks travelling along relativistic jets. So far only very simple quasi-one dimensional models have been used to study this phenomenon. Recent progress in numerical methods for relativistic fluid dynamics (Falle \& Komissarov 1995) make it possible to carry out much more realistic simulations of such flows.

Some superluminal sources (e.g. 4 C39.25, Shaffer et al. 1987) have stationary knots as well as moving ones. Daly \& Marcher (1988) proposed that these stationary knots could be identified with steady shocks, which would arise quite naturally if the jet is reconfined. Where the relative proximity and brightness of the compact radio sources allow us to observe their parsec scale jets in detail, they often reveal peculiar limb-brightening (e.g. in M87, Reid et al. 1989 and 3C345, Unwin 1993). This may be due to a turbulent boundary layer (Komissarov 1990) but it could also be caused by a reconfinement shock close to the jet boundary.

In this paper we use numerical simulations to study the combined effect of the quasi-stationary reconfinement shock and moving shocks due to variations in the central source on the appearance of a relativistic jet.

We first set up a steady flow in which the jet propagates through an external medium whose pressure decreases like $z^{-1.9}$ where $z$ is the distance from the source. We then allow the jet velocity at the source to vary in such a way that shocks are produced in the gas as soon as it emerges from the source.

The radio emission is determined by assuming that both the magnetic and relativistic pressures are proportional to the thermal pressure. The relativistic version of the radiative transfer equation (Mihalas 1986) is then used to obtain the intensity as seen by a distant observer. 


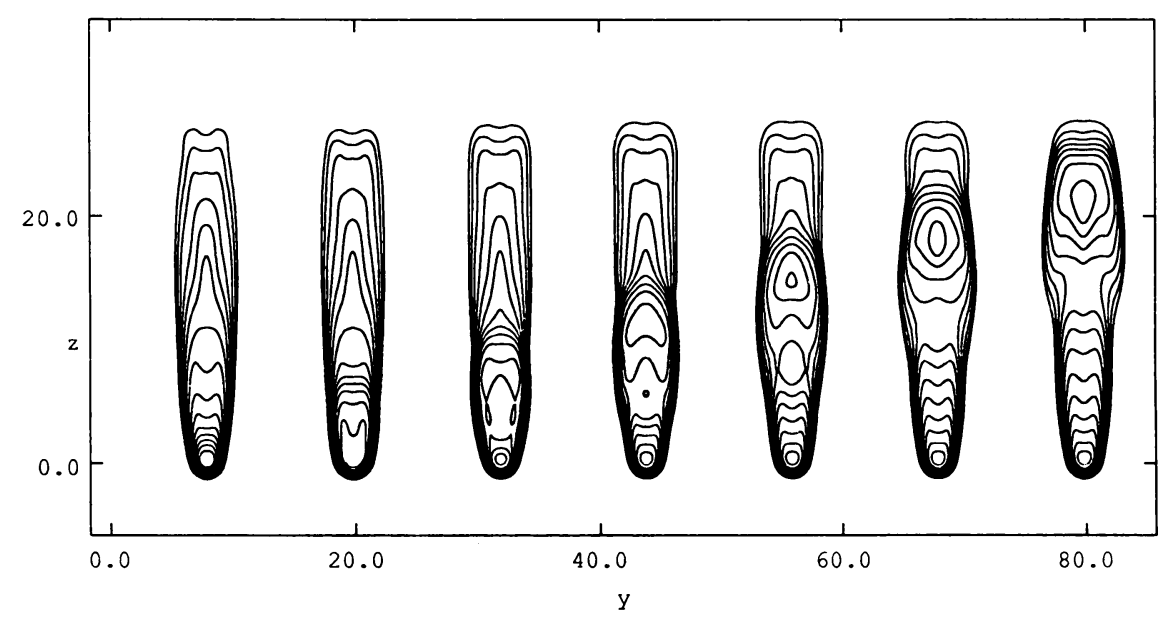

Figure 1. The figures show logarithmic contours of brightness distribution with a dynamic range of 1000:1 at unit time intervals. Note that the speed of light is unity. The images are smoothed with a gaussian beam of size 0.5 .

Figure 1 shows the results for an inclination angle of $20^{\circ}$. The most striking property of the images is that they show only one moving knot, whereas there are actually eight travelling shocks in the flow. This is a result of relativistic retardation just like the superluminal motion itself. The apparent speed of the knot is $V_{a p p} \simeq 4 c$, which agrees very well with the analytic expression (Begelman et al. 1984).

Another interesting feature of these maps is the absence of a stationary knot. However, the reconfinement shock is the cause of the limb-brightening in the first and the last thirds of the jet image and the center brightening in the middle. One can also clearly see that the moving knot is limb-brightened when going through the limb-brightened region of the underlying flow. It is interesting that knot $\mathrm{N} 2$ in the M87 jet does indeed reveal this kind of structure (Reid et al.,1987).

\section{References}

Begelman M.C., Blandford R.D., Rees M.J., 1984, Rev.Mod.Phys., 56, 255.

Daly R.A., Marscher A.P., 1988, ApJ., 334, 539.

Falle S.A.E.G., Komissarov S.S., 1995, Mon.Not.R.astr.Soc., in press.

Komissarov S., 1990, Soviet Astron. Lett., 16(4), 284.

Mihalas D., 1986, in Winkler K.-H. A. \& Norman M. L., eds, Astrophysical Radiational Hydrodynamics, Reidel, Dordrecht, p.61.

Reid M.J., Biretta J.A., Junior W., Maxlow T., Spencer R.E., 1989, Ap.J., 336, 112.

Shaffer et al., 1987, Ap.J., 314, L1.

Unwin R.J., 1993, in Davis R.J. \& Booth R.S., eds, Sub-arcsecond Radio Astronomy, Cambridge University Press, Cambridge, p.189. 